\title{
Numerical investigation of heated gas flow in a thermoacoustic device
}

\author{
Kazuto Kuzuu * and Shinya Hasegawa \\ Department of Prime Mover Engineering, Tokai University, Hiratsuka, Kanagawa \\ 2591292, Japan \\ * Corresponding author. Tel.: +81-463584872; fax: +81-463598293. \\ E-mail address: kuzuu@tokai-u.jp,s.hasegawa@tokai-u.jp
}

Self-sustained oscillation in a standing wave thermoacoustic device is reproduced via computational fluid dynamics simulations, and heated flow behavior in the device is explored using the results obtained. The straight-type thermoacoustic device is composed of two resonance tubes, two heat exchangers and a stack. In these simulations, both the acoustic characteristics and the temperature field during self-sustained oscillation are considered. Therefore, to reproduce the self-sustained oscillatory flow, an acoustic signal is injected into the computational domain as a trigger pulse. From the results, oscillatory fluid motion around the engine is investigated, and characteristics, including the acoustic field or work flow, the energy dissipation, the work source and other associated aspects, are estimated. The results agree well with those of linear theory, although high energy dissipation caused by vortex generation is observed near the engine. This result verifies the computational fluid dynamics simulation results. The temperature field around the engine is also investigated. The results show occurrence of asymmetrical temperature oscillations within the heat exchangers. This behavior cannot be predicted using linear theory because the non-uniform temperature gradient in the engine unit is 
transferred in stream-wise by convection. Finally, a modification to conventional linear theory is suggested to reproduce this behavior.

Keywords: Thermoacoustics, Heat exchanger, Computational fluid dynamics, Oscillatory flow 


\section{Introduction}

The thermoacoustic device reviewed by Swift in 1988 combines the advantages of the inherent thermal efficiency properties of the Stirling cycle with the ability to work using a minimum number of moving parts [1]. Swift et al. demonstrated the feasibility of the device using an inexpensive prototype [2], and since then, thermoacoustic devices have attracted considerable attention as applications of renewable heat energy. Many basic and practical studies of these devices have been performed in recent years.

Thermoacoustic devices work using thermoacoustic phenomena. Theories to explain these phenomena were originally formed based on acoustic theory [3-7], and the linear theories developed by Rott [8,9] and Tijdeman [10] are still commonly applied to developments in these devices. For example, the Design Environment for Low-amplitude Thermoacoustic Engines (DELTAE), which is a numerical analysis code developed by Swift et al. [11], uses the linear theory of Rott [8] and is useful for thermoacoustic device design.

Linear theory is thus significant when dealing with thermoacoustic phenomena that occur in a thermoacoustic device. However, some phenomena cannot currently be explained using linear theory. It is not actually possible to predict non-linear phenomena like two or three-dimensional vortex generation. Heat transfer in the heat exchanger is another problem to be solved in this field, and the problem must be studied using methods beyond linear theory. Many studies of heat transfer in thermoacoustic devices have been performed recently, and they can be classified into several different types of approaches.

The first approach combines linear theory and numerical calculations. Piccolo et al. introduced a simple energy conservation model coupled with classical linear thermoacoustic theory, and used it to estimate the heat transfer properties of thermoacoustic heat exchangers 
composed of several parallel plates $[12,13]$. In another method, Jong et al. proposed a heat transfer model for one-dimensional oscillatory flow. This model can be applied to parallel-plate thermoacoustic heat exchangers, and was used to investigate their heat transfer properties [14].

Experimental approaches can also be used to investigate heat transfer. Keolian et al. investigated the heat transfer of parallel-plate heat exchangers in oscillatory flow environments [15]. They estimated heat exchanger effectiveness based on its temperature field, which was measured using thermistor probes placed at the heat exchanger exits and entrances. They also compared the results of their study with those obtained using the DELTAE code [11]. Additionally, Jaworski et al. investigated the heat transfer properties of parallel-plate heat exchangers through acetone-based planar laser-induced fluorescence (PLIF) measurements [16,17], and compared their measured results with numerical results [18,19].

The final approach is based on computational fluid dynamics (CFD). This approach is advantageous because the assumptions in linear theory are excluded, and because the heat transfer properties of a thermoacoustic device can be calculated directly from its temperature field. Some numerical simulations of oscillatory flow in heated pipes were performed by Cheng et al. [20,21] While their simulations were for oscillatory flow induced by acoustic wave propagation, their results provide the characteristic features of an oscillatory temperature field in the tube. In another numerical simulation, Cao et al. investigated the energy flux density in a thermoacoustic couple under acoustic standing wave conditions [22] In this study, they estimated the effects of the displacement amplitude on heat transfer. The study is significant for heat transfer estimation because the displacement amplitude in Rott's theory $[8,9]$ is assumed to be negligible when compared with the device length. Ishikawa et al. also studied the flow fields and energy transport near thermoacoustic couples through numerical simulations using a $2 \mathrm{D}$ full 
Navier-Stokes solver [23]. Marx et al. performed numerical simulations of a thermoacoustic refrigerator that consisted of a resonator and a parallel plate stack [24]. They compared their results with those predicted by linear theory, and showed that there is a difference in mean temperature between the fluid and the plate. Jaworski et al. also investigated the oscillatory flow and heat transfer of parallel heat exchangers, and compared their results with experimental data [25]. Using both numerical results and experimental data, they demonstrated the effect of the temperature field on oscillatory flow and the dependencies of heat transfer on the Reynolds number.

Additionally, self-sustained oscillation is also reproduced and discussed with respect to CFD simulations of thermoacoustic phenomena. Hantschk et al. simulated self-sustained oscillation in a Rijke tube [26]. While the simulated tube includes only heating elements, rather than heat exchangers, they discussed non-linearity in the heat transfer process. Recently, other CFD simulations of self-sustained oscillation in thermoacoustic engines have been performed. Spoelstra et al. simulated a travelling-wave thermoacoustic engine, and showed strong non-linear effects for high-amplitude thermoacoustic systems [27]. Zink et al. showed the transition from initial disturbance to self-sustained oscillation in a thermoacoustic engine, and explored the effects of a curved resonator [28]. Dai et al. simulated self-sustained oscillation in a $300 \mathrm{~Hz}$ standing wave thermoacoustic engine [29] by visualizing flow fields at the ends of the stack, and discussed multi-dimensional effects that occurred because of the abrupt change in flow area.

As described above, many studies of thermoacoustic devices have been performed using a variety of approaches. CFD simulations attract particular attention because of their ability to reproduce non-linear effects in thermoacoustic phenomena, and the technique has progressed such that the non-linearity of flow behavior in self-sustained oscillation can be discussed. 
However, while the acoustic fields obtained from these simulations are comparable with those obtained using conventional linear theory, the non-linearity of heated flow behavior, which must affect the system heat transfer, has not been discussed adequately to date.

In this study, to investigate such heated flow behavior occurring in a thermoacoustic device in greater detail, unsteady CFD simulations of self-sustained oscillatory flow are performed. For this purpose, a full-scale device model was set up and the engine unit was given a suitable temperature profile. Using this setup, self-sustained thermoacoustic oscillation can be reproduced numerically. A linear analysis based on conventional thermoacoustic theory is performed simultaneously, and the results are compared. In particular, rather than simply compare the results for the acoustic characteristics, the relationship between the acoustic characteristics when treated using linear theory and the flow fields obtained from CFD is clarified, and the linear and

non-linear characteristics of oscillatory flows are discussed. Finally, the temperature field behavior that cannot be explained using linear theory is investigated.

\section{Calculations}

\subsection{Calculation method in $C F D$}

CFD simulations are carried out using the LS-FLOW unstructured compressible flow solver developed by the Japan Aerospace eXploration Agency (JAXA) [30]. The solver is based on three-dimensional unsteady compressible Navier-Stokes equations. The basic equations are as follows.

$$
\frac{\partial}{\partial t} \oiiint_{\mathcal{V}} Q d \mathcal{V}+\oiint_{S}\left(F_{e}-F_{v}\right) d S=0
$$




$$
\begin{aligned}
& Q=\left[\begin{array}{c}
\rho \\
\rho u_{x} \\
\rho u_{y} \\
\rho u_{z} \\
E
\end{array}\right], \quad F_{e}=\left[\begin{array}{c}
\rho U \\
\rho u_{x} U+n_{x} p \\
\rho u_{y} U+n_{y} p \\
\rho u_{z} U+n_{z} p \\
(E+p) U
\end{array}\right], \quad F_{v}=\left[\begin{array}{c}
0 \\
\tau_{x x} n_{x}+\tau_{y x} n_{x}+\tau_{z x} n_{x} \\
\tau_{x y} n_{y}+\tau_{y y} n_{y}+\tau_{z y} n_{y} \\
\tau_{x z} n_{z}+\tau_{y z} n_{z}+\tau_{z z} n_{z} \\
\left(u_{i} \tau_{i j}-q_{j}\right) n_{j}
\end{array}\right] \\
& U=u_{x} n_{x}+u_{y} n_{y}+u_{z} n_{z}, \quad u_{i}=\left[\begin{array}{c}
u_{x} \\
u_{y} \\
u_{z}
\end{array}\right], \quad \tau_{i j}=-\frac{2}{3} \mu(\nabla \cdot U) \delta_{i j}+\mu\left(\frac{\partial u_{i}}{\partial x_{j}}+\frac{\partial u_{j}}{\partial x_{i}}\right) \\
& E=\frac{1}{\gamma-1} p+\frac{1}{2} \rho U^{2}, \quad q_{i}=-\lambda \nabla T,
\end{aligned}
$$

where $\rho, u_{i}, p, T, \mu$ and $\lambda$ are the density, the velocity vector, the pressure, the temperature, the viscosity and the heat conductivity of the gas, respectively, and $n_{i}$ is the vector normal to the volume element surface. The main numerical schemes used in this simulation are given in Table 1.

Table 1. Numerical schemes used in CFD simulations.

\begin{tabular}{ccc}
\hline \hline & Calculation & Name of scheme \\
\hline & Time integration & Three points backward step approximation \\
\hline Implicit solution & LU-SGS [31] \\
\hline \multirow{2}{*}{ Numerical flux } & Interpolation & MUSCL scheme by Green-Gauss method \\
& Convective term & SLAU [32] \\
& Viscous term & Wang's method [33] \\
\hline \hline
\end{tabular}

\subsection{CFD calculation model}

Figure 1 shows the calculation model used in this simulation. The engine unit has three components: hot and cold heat exchangers (HEX and CEX), and a stack (STK). Each component contains six flat plates and seven channels. The computational domain includes the buffer region. The individual part sizes and boundary conditions are shown in Fig. 1(a). The model is twodimensional; this is achieved by providing symmetrical conditions in the $\mathrm{z}$ direction. The wall temperatures, excluding those of the six flat plates of the engine unit, are all fixed at room temperature $(298.15 \mathrm{~K})$. The temperatures in the engine unit are $423.15 \mathrm{~K}$ for HEX and 298.15 K 
for CEX. A linear distribution ranging between $423.15 \mathrm{~K}$ and $298.15 \mathrm{~K}$ is used for the STK temperature. The working gas is air, the working pressure is $p=1.01325 \times 10^{5} \mathrm{~Pa}$, and the temperature $T=298.15 \mathrm{~K}$.

In this simulation, to induce self-sustained oscillatory flow, the pressure disturbance was injected as a trigger pulse from the open end. The simulations actually start from a static initial condition and continue for a short time. Then, one-half cycle of a sinusoidal acoustic wave is injected as a disturbance with $\hat{p} \approx 283 \mathrm{~Pa}$ and $f=21.2 \mathrm{~Hz}$ from the open end into the buffer region. The supply of this acoustic wave is then terminated. 
$\mathrm{BC}$

Upper \& Lower Wall, Closed end, Wall of Buffer Region

: non-slip \& $\mathrm{Tw}=298.15 \mathrm{~K}$

HEX : non-slip \& Tw $=423.15 \mathrm{~K}$

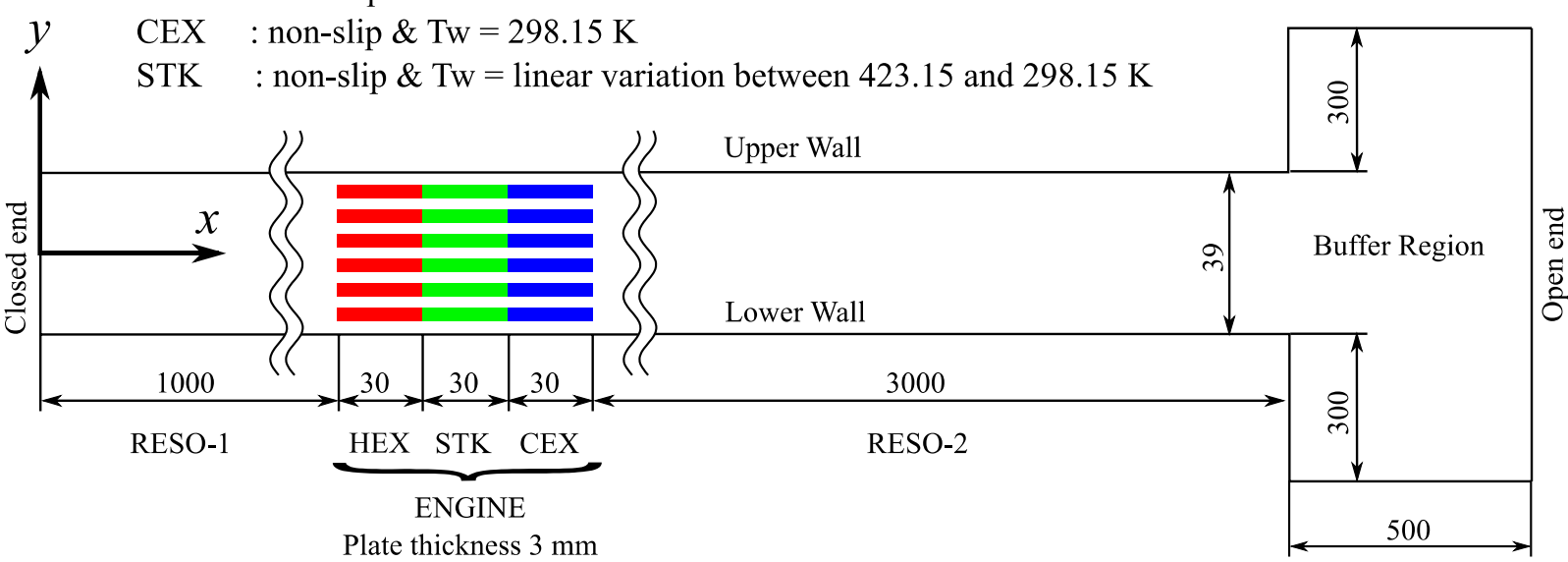

Channel width $3 \mathrm{~mm}$

(a) Domain, size of each component and boundary conditions

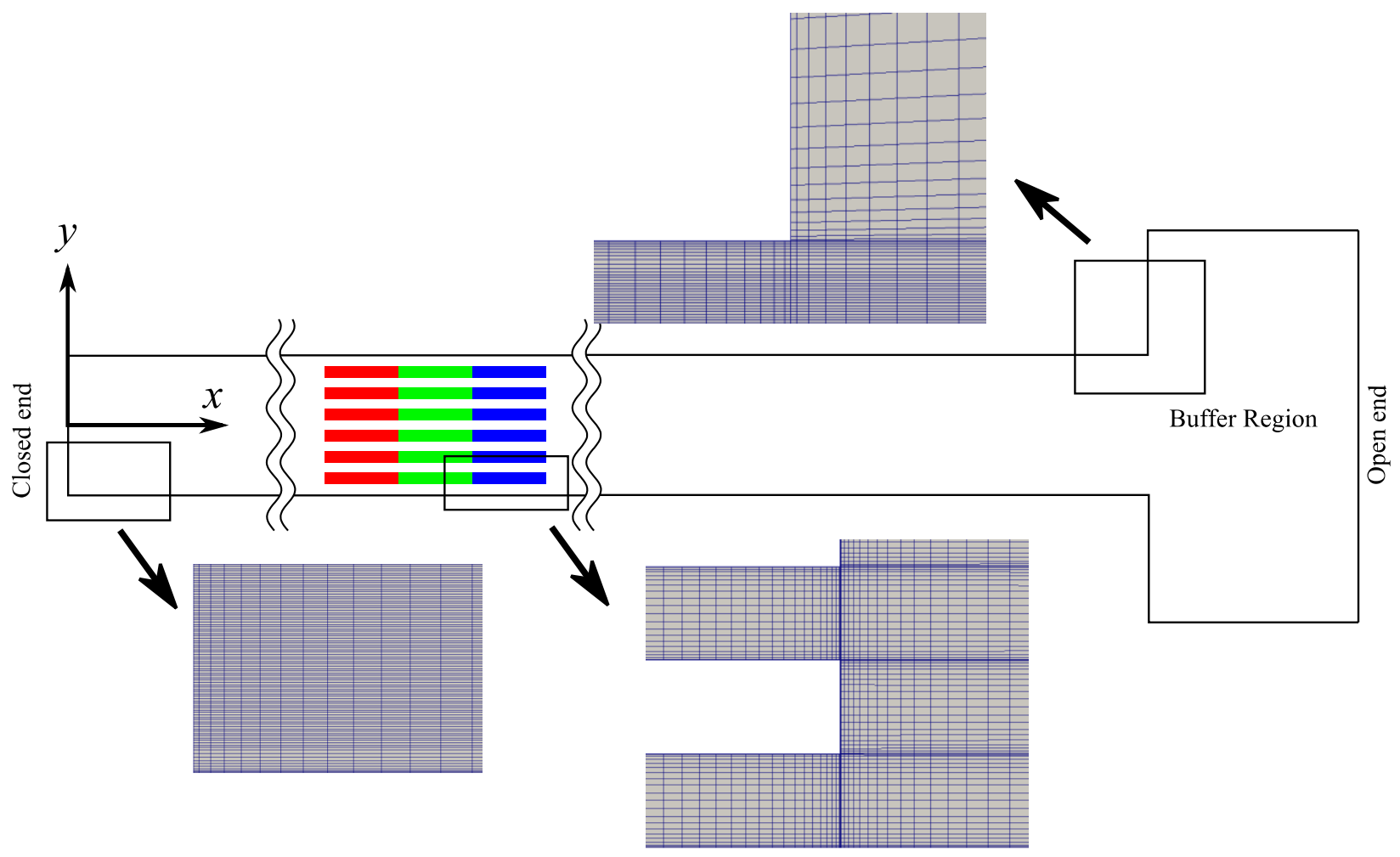

(b) Enlarged mesh configuration of each part

Fig. 1. Computational domain, boundary conditions and mesh configuration. 
The mesh configuration is composed of hexahedral cells that were converted from nonuniform Cartesian grids. The concentrated mesh state of the representative parts is shown in Fig. 1(b). For two-dimensional calculations, the number of divisions in the z-direction is set at one. The total number of cells is approximately 300000, and the minimum mesh size, which corresponds to the distance from the boundary wall to the adjacent mesh, is $0.0237 \mathrm{~mm}$. The time step used for this simulation is $2.0 \mu$ s, which corresponds to a Courant-Friedrichs-Lewy (CFL) number of 30 based on the sound velocity.

\subsection{Numerical calculations based on linear theory}

Using Rott's linear theory [8], the basic equations for one-dimensional thermoacoustic oscillatory flow within a tube can be developed and can thus be expressed as simultaneous differential equations with respect to velocity and pressure.

$\frac{\partial p_{1}}{\partial x}=-\frac{j \omega \rho_{m}}{\left(1-\chi_{v}\right)}\langle u\rangle_{r}$

$\frac{\partial\langle u\rangle_{r}}{\partial x}=-\frac{j \omega}{p_{m}}\left[1-\frac{\gamma-1}{\gamma}\left(1-\chi_{v}\right)\right] p_{1}+\frac{\chi_{\alpha}-\chi_{v}}{\left(1-\chi_{v}\right)(1-\sigma)} \frac{1}{T_{m}} \frac{\partial T_{m}}{\partial x}\langle u\rangle_{r}$,

where $\chi_{v}$ and $\chi_{\alpha}$ are the viscous and thermal thermoacoustic functions, respectively, and have two-dimensional formulations as follows.

$\chi_{v}=\frac{\tanh \left((1+j) \sqrt{\omega \tau_{v}}\right)}{(1+j) \sqrt{\omega \tau_{v}}}, \quad \chi_{\alpha}=\frac{\tanh \left((1+j) \sqrt{\omega \tau_{\alpha}}\right)}{(1+j) \sqrt{\omega \tau_{\alpha}}}$

$\tau_{v}=r_{0}^{2} / 2 v, \quad \tau_{\alpha}=r_{0}^{2} / 2 \alpha$

Here, $r_{0}$ is one-half of the two-dimensional channel width.

These equations are calculated numerically. For example, Ueda et al. proposed a numerical method based on the fourth-order Runge-Kutta method and calculated the critical temperature ratio for self-sustained thermoacoustic oscillation [34]. 
In the linear analysis, the pressure amplitude at the antinode point (closed end) is given as a boundary condition. A value of $495 \mathrm{~Pa}$ is calculated using CFD. For the temperature conditions, the time and section-averaged temperature obtained from CFD is used. These conditions lead to the properties shown in Table 2 .

Table 2. Properties of air within the engine.

\begin{tabular}{cccc}
\hline \hline$T(\mathrm{~K})$ & $\rho\left(\mathrm{kg} / \mathrm{m}^{3}\right)$ & $v\left(\mathrm{~m}^{2} / \mathrm{s}\right)$ & $\tau_{v} \omega$ \\
\hline 402 & 0.8782 & $2.6299-05$ & 5.617 \\
298.15 & 1.1847 & $1.5561-05$ & 9.493 \\
\hline \hline
\end{tabular}

\section{Results and discussion}

\subsection{Production of self-sustained oscillation}

To confirm self-sustained oscillation, time variation of the physical values after termination of the trigger injection is observed. Figure 2 shows the variations in the axial velocity and pressure at $(x, y)=(1.04,0.0)$. The acoustic signal injection time is $t=2.054$ to $2.078 \mathrm{~s}$. As shown in the figure, the phenomenon is considered to be in a periodic steady state after $4 \mathrm{~s}$ and is then in self-sustained oscillation. 


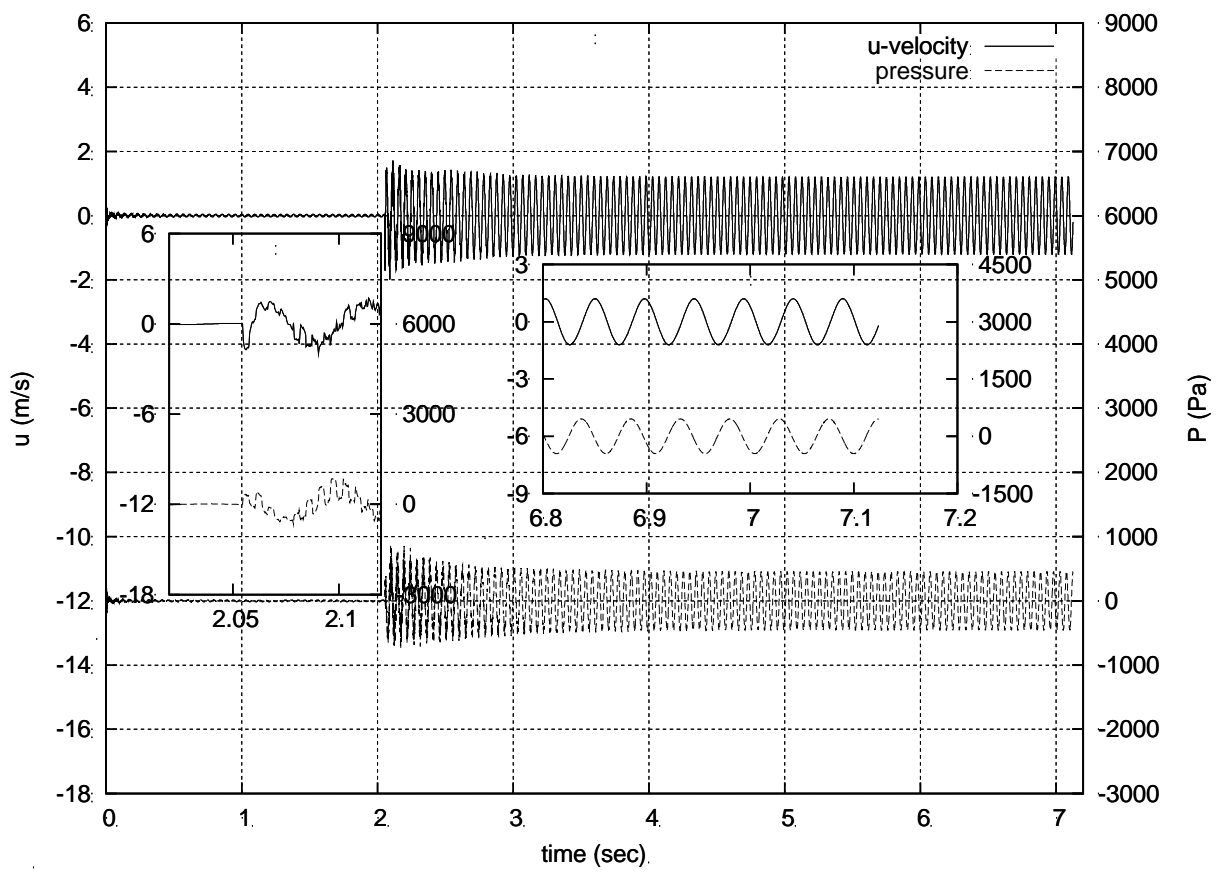

Fig. 2. Time variation of velocity and pressure amplitude at $(x, y)=(1.04,0.0)$.

The enlarged graph shows the period around the occurrence of the trigger pulse and the periodic steady state.

\subsection{Verification of $C F D$}

Oscillatory flow behavior in the device is investigated, and the CFD results are compared with those from linear theory. Here, we show the velocity profiles at two device cross-sections. The velocity profile of oscillatory flow varies with time. Figure 3 shows 12 phases in a single cycle. In Fig. 3, the velocity amplitude is the section-averaged value at the midpoint of the STK $(x=1.045 \mathrm{~m})$, and the corresponding displacement amplitude is also shown. 


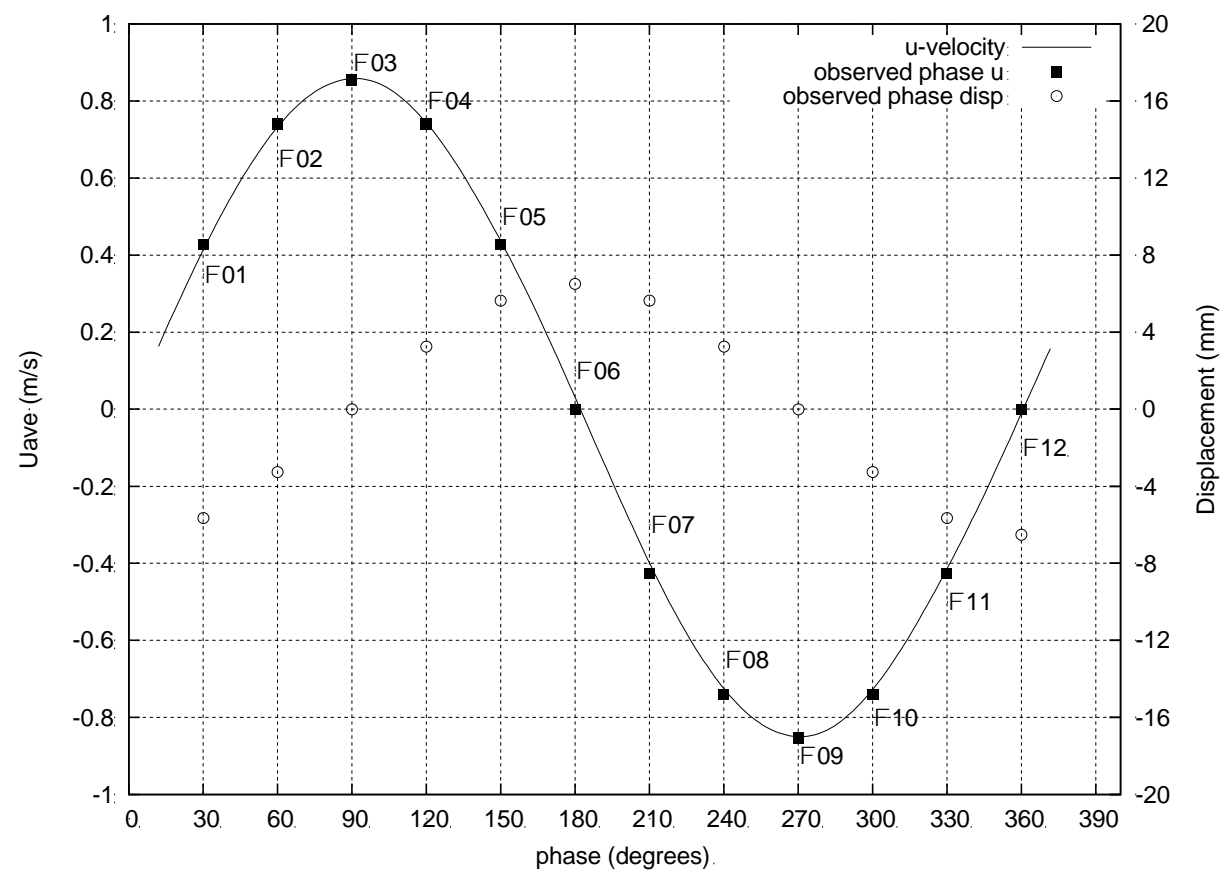

Fig. 3. Phase variations of section average velocity and displacement amplitude at $x=1.045 \mathrm{~m}$ (mid-section of the STK).

Figure 4 shows the variations in the velocity profiles. Each graph corresponds to profiles on the section including the STK and the resonance tube $(x=1.045$ and $2.00 \mathrm{~m})$. Both profiles agree with the results of linear theory. This implies that the fundamental oscillatory flow behavior obeys linear theory within the device. 

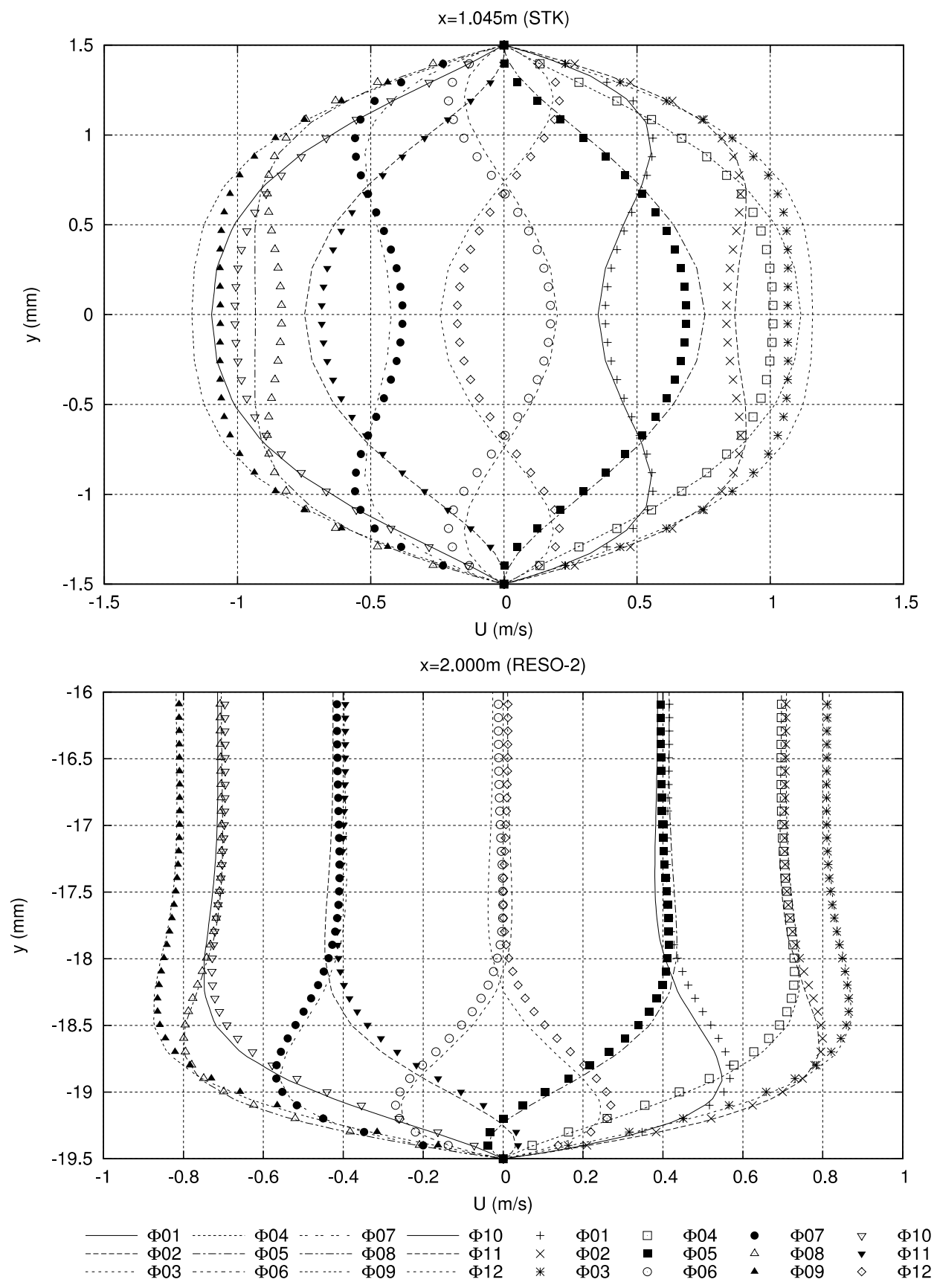

Fig. 4. Comparisons of velocity profiles.

(Lines: CFD; symbols: linear analysis) 


\subsection{Acoustic properties}

In thermoacoustic theory, the acoustic power is expressed as a work flow. From the CFD results, we can calculate this value using Eq.(5).

$I=\frac{\omega}{2 \pi} \times \int_{t}^{t+2 \pi / \omega}\left\{\oiint_{A}\left(p-p_{m}\right) u_{x} d A\right\} d t$

In Eq. (5), the work flow, $I$, is the time-averaged value over a single acoustic wave cycle.

Figure 5 shows a comparison of the CFD simulation results with those from linear analysis. Here, the temperature gradient used in the linear analysis is based on the CFD results. In the figure, the CFD results almost agree with the power gain obtained in the linear analysis. However, the CFD power gain is approximately $9 \%$ smaller than that determined by linear analysis. This may be caused by differences between the CFD simulations and the linear analysis in terms of temperature conditions at each section. In fact, while the temperature is assumed to be common at each section of the engine channels in the linear analysis, the temperature differs in every channel in the CFD simulations. This difference is described in detail later. 


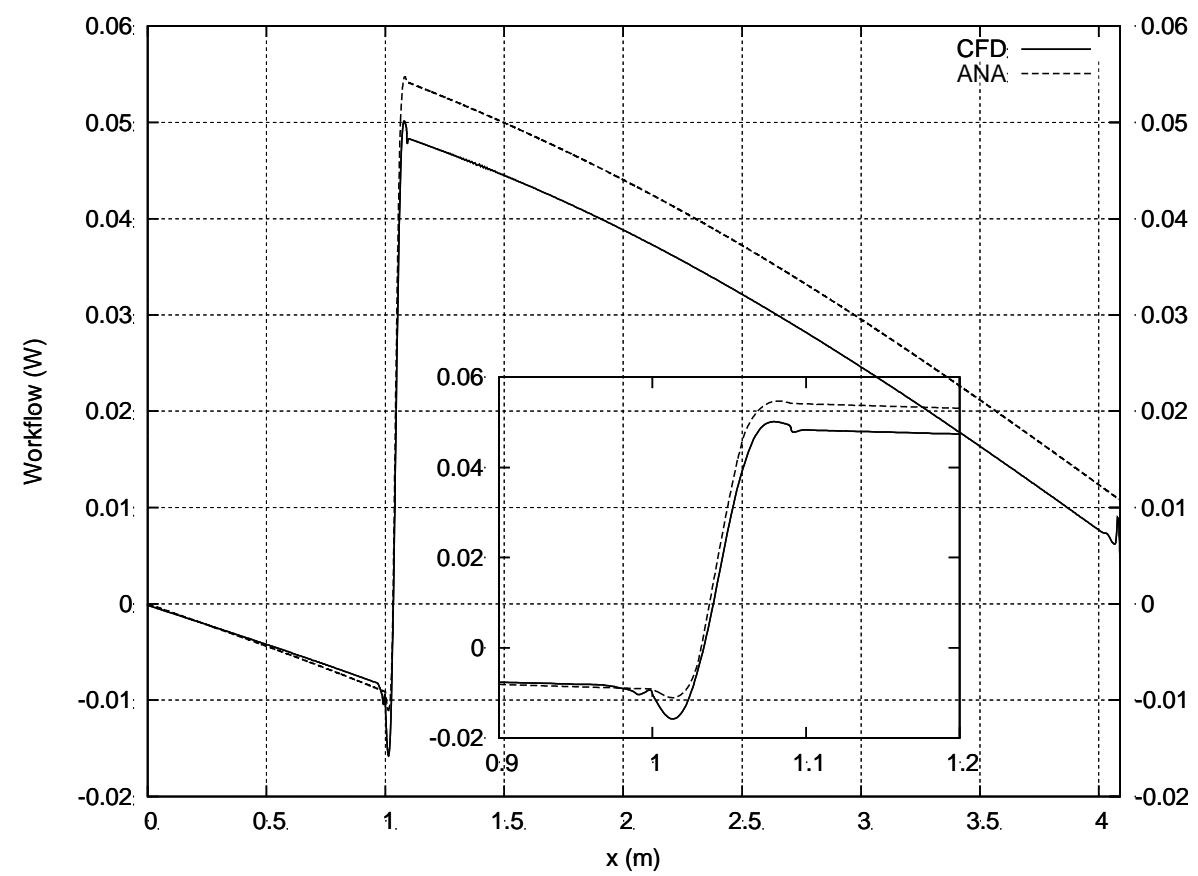

Fig. 5. Comparison of work flow characteristics between CFD and linear analysis.

The enlarged graph shows the characteristics around the engine unit.

\subsection{Energy dissipation and work source}

In this section, we calculate the energy dissipation and work source from the CFD results and compare them with the results from linear theory.

First, we consider the energy dissipation and the work source as thermoacoustic properties. To explain the cause of the work flow, Tominaga [35] introduced the concept of energy dissipation and the work source based on linear theory of flow in a tube. In this theory, energy dissipation is classified in terms of the kinetic and potential energy dissipations, or $W_{v}$ and $W_{p}$, respectively. Additionally, the work source is divided into two parts, i.e., the traveling and standing wave components, $W_{\text {prog }}$ and $W_{\text {stand }}$, respectively.

These parameters are defined as follows.

$W_{v}=-\frac{A}{2} \operatorname{Re}\left[\frac{j \omega \rho_{m}}{\left(1-\chi_{v}\right)}\right]\left|\langle u\rangle_{r}\right|^{2}$ 
$W_{p}=-\frac{A}{2} \operatorname{Re}\left[\frac{j \omega}{\gamma p_{m}}\left(1+(\gamma-1) \chi_{\alpha}\right)\right]\left|p_{1}\right|^{2}$

$W_{\text {prog }}=\frac{A}{2} \operatorname{Re}\left[\frac{\left(\chi_{\alpha}-\chi_{v}\right)}{\left(1-\chi_{v}\right)(1-\sigma) T_{m}}\right]\left|p_{1}\right|\left|\langle u\rangle_{r}\right| \cos \varphi_{p u} \frac{\partial T_{m}}{\partial x}$

$W_{\text {stand }}=\frac{A}{2} \operatorname{Im}\left[\frac{\left(\chi_{\alpha}-\chi_{v}\right)}{\left(1-\chi_{v}\right)(1-\sigma) T_{m}}\right]\left|p_{1}\right|\left|\langle u\rangle_{r}\right| \sin \varphi_{p u} \frac{\partial T_{m}}{\partial x}$

Here, we must extract the energy dissipation and the work source from the CFD results.

The first law of thermodynamics is commonly given as

$\delta Q=\delta e_{i}+p \delta v$

$\delta Q$ is the heat energy added to the fluid element from an external system, $\delta e_{i}$ is the increment in the internal energy, and $p \delta v$ corresponds to the work in which the element acts for the external system. Given that $\delta Q$ is the heat energy added to the fluid element by heat conduction, the energy equation that applies to two-dimensional fluid motion is obtained as follows.

$$
\begin{gathered}
\rho \frac{D e_{i}}{D t}=-\left\{\frac{\partial}{\partial x}\left(-k \frac{\partial T}{\partial x}\right)+\frac{\partial}{\partial y}\left(-k \frac{\partial T}{\partial y}\right)\right\}+(-p)\left(\frac{\partial u}{\partial x}+\frac{\partial v}{\partial y}\right) \\
+\left(\tau_{x x} \frac{\partial u}{\partial x}+\tau_{y x} \frac{\partial u}{\partial y}+\tau_{x y} \frac{\partial v}{\partial x}+\tau_{y y} \frac{\partial v}{\partial y}\right)
\end{gathered}
$$

In the above equation, the third term corresponds to kinetic energy dissipation by viscosity, while the second term can be regarded as a summation of the potential energy dissipation and the work source. By integrating these terms with respect to both time and cross-section, the energy dissipation and the work source properties can be obtained as follows. 
$\underline{\text { Kinetic energy (viscous) dissipation }}$

$$
\begin{aligned}
& \phi=\tau_{x x} \frac{\partial u}{\partial x}+ \tau_{y x} \frac{\partial u}{\partial y}+\tau_{x y} \frac{\partial v}{\partial x}+\tau_{y y} \frac{\partial v}{\partial y} \\
&=\mu \\
& \times\left(2\left(\left(\frac{\partial u}{\partial x}\right)^{2}+\left(\frac{\partial v}{\partial y}\right)^{2}\right)+\left(\frac{\partial v}{\partial x}+\frac{\partial u}{\partial y}\right)^{2}-\frac{2}{3}\left(\frac{\partial u}{\partial x}+\frac{\partial v}{\partial y}\right)^{2}\right) \\
&\langle\phi\rangle_{t}=\frac{\omega}{2 \pi} \times \int_{t}^{t+2 \pi / \omega}\left\{\oiint_{A} \phi d A\right\} d t
\end{aligned}
$$

$\underline{\text { Summation of potential energy dissipation and work source }}$

$$
\begin{gathered}
\psi=-\left(p-p_{a}\right) \times\left(\frac{\partial u}{\partial x}+\frac{\partial v}{\partial y}\right) \\
=\frac{\left(p-p_{a}\right)}{\rho} \times \frac{D \rho}{D t} \\
\langle\psi\rangle_{t}=\frac{\omega}{2 \pi} \times \int_{t}^{t+2 \pi / \omega}\left\{\oiint_{A} \psi d A\right\} d t
\end{gathered}
$$

Figure 6 shows the energy dissipations and the work source calculated using the above equations. The work source in the resonance tube can be neglected because of the lack of a heat source. From this, it can be said that both the viscous and potential energy dissipations almost follow linear theory in the resonance tube.

Additionally, we show the kinetic energy dissipation within and near the engine. In CFD, this dissipation is calculated using Eq. (12). The enlarged graph in Fig. 6 shows a comparison of the results of CFD and linear analysis. The graph shows good agreement, but differences can be observed near the heat exchanger extremities $(x=1.00$ and $1.09 \mathrm{~m})$. This may be the effect of vortex generation around the corners of the engine plates. However, the work flow is not so 
strongly affected by this difference because the total energy loss is decided by the integration in the $\mathrm{x}$-direction and the peak value is limited to quite a narrow area.

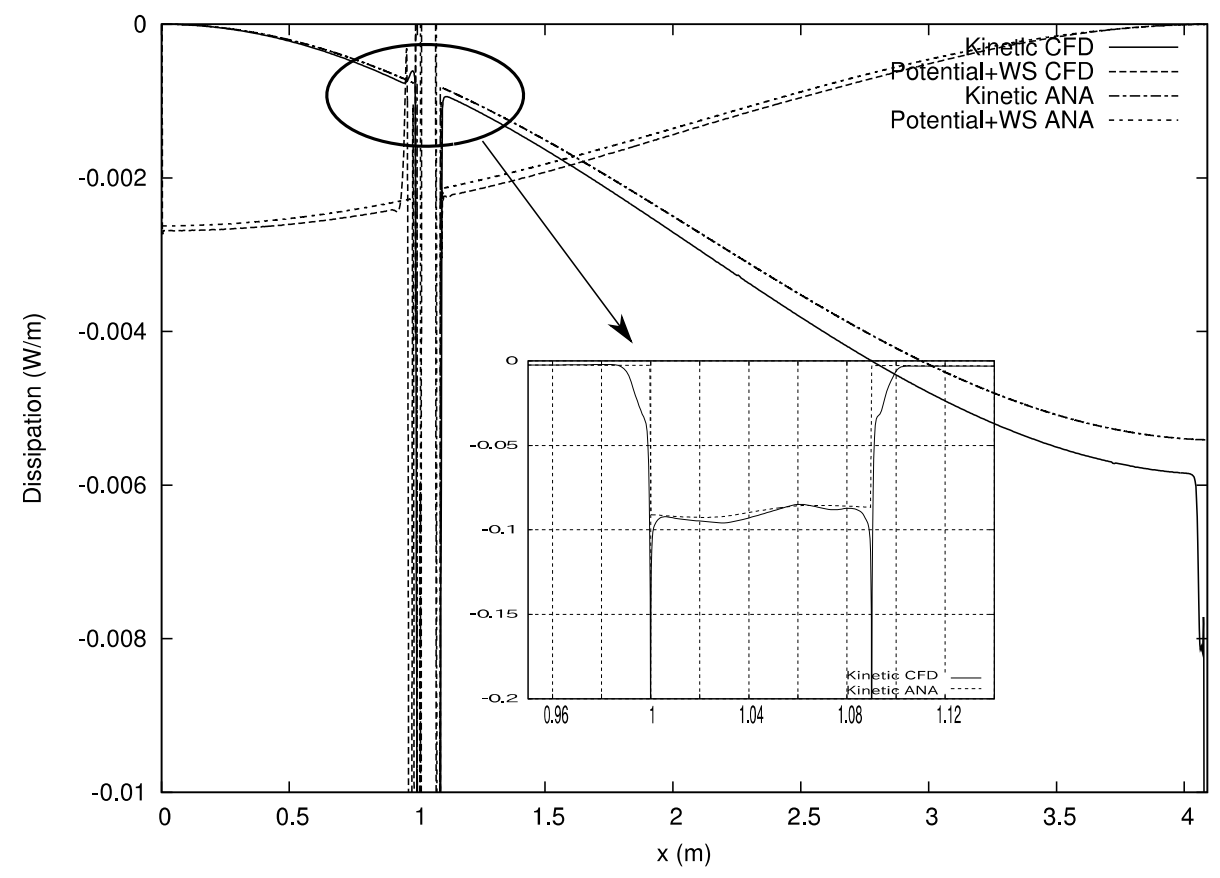

Fig. 6. Comparison of dissipation characteristics from CFD and linear analysis.

Next, the effects of potential energy dissipation and the work source around the engine unit are investigated. In CFD, this effect is described using Eq. (14), and is the summation of the potential energy dissipation and the work source. Therefore, the analytical results for comparison must be expressed using the summation of $W_{p}, W_{\text {prog }}$ and $W_{\text {stand }}$ from Eqs. (7)-(9). Figure 7 compares the results from CFD and linear theory. They show almost perfect agreement. 


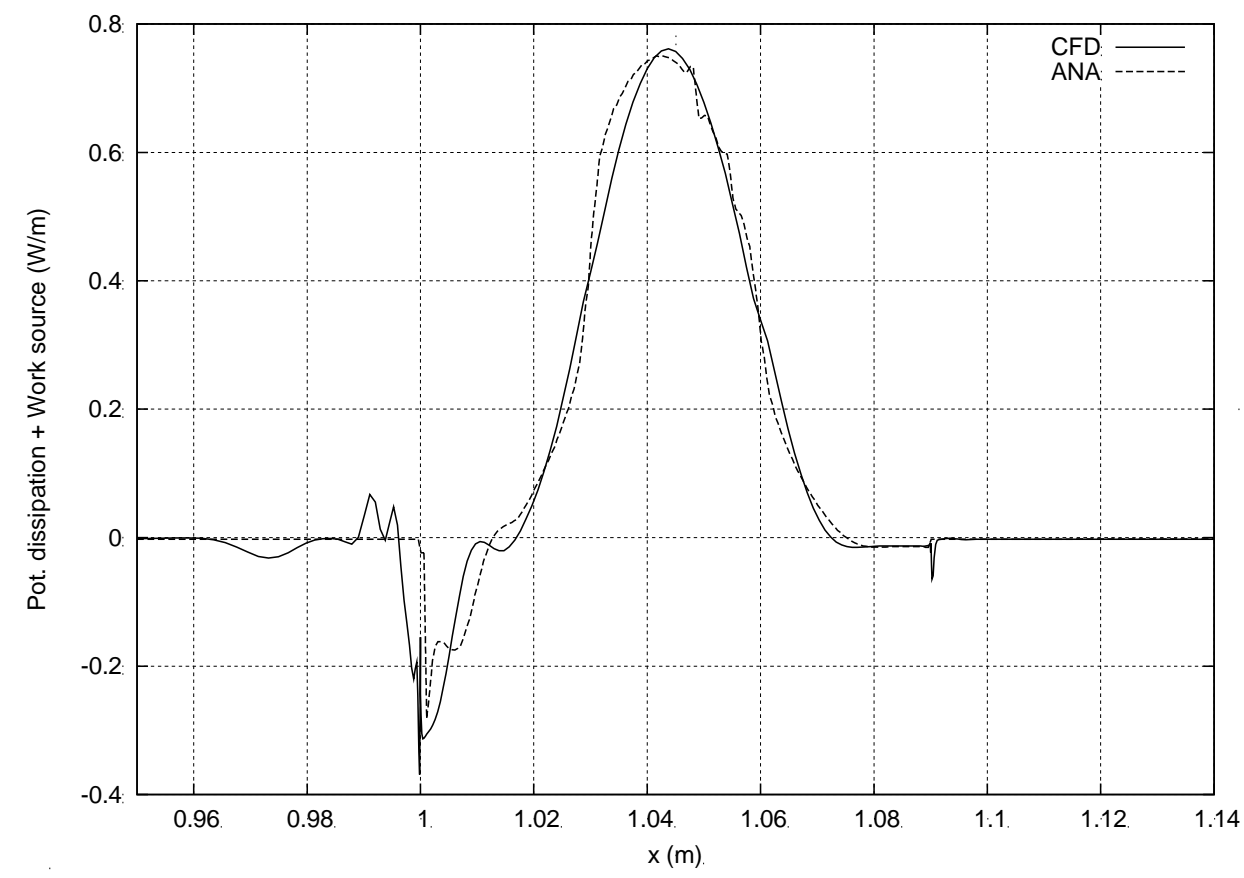

Fig. 7. Effects of potential energy dissipation and the work source.

Compared values: $\langle\psi\rangle_{t}$ in CFD and $W_{p}+W_{\text {prog }}+W_{\text {stand }}$ in the analysis.

From thermoacoustic theory in a tube, the work flow must be consistent with the integration of the above values, as follows.

$I=\int_{0}^{x}\left(W_{v}+W_{p}+W_{\text {prog }}+W_{\text {stand }}\right) d x$

Figure 8 compares the results of the integration in Eq. (16) above with the work flow calculated from Eq. (5), and shows good agreement between these results. The work source and the dissipation are thus considered to have been correctly estimated using the CFD flow data. 


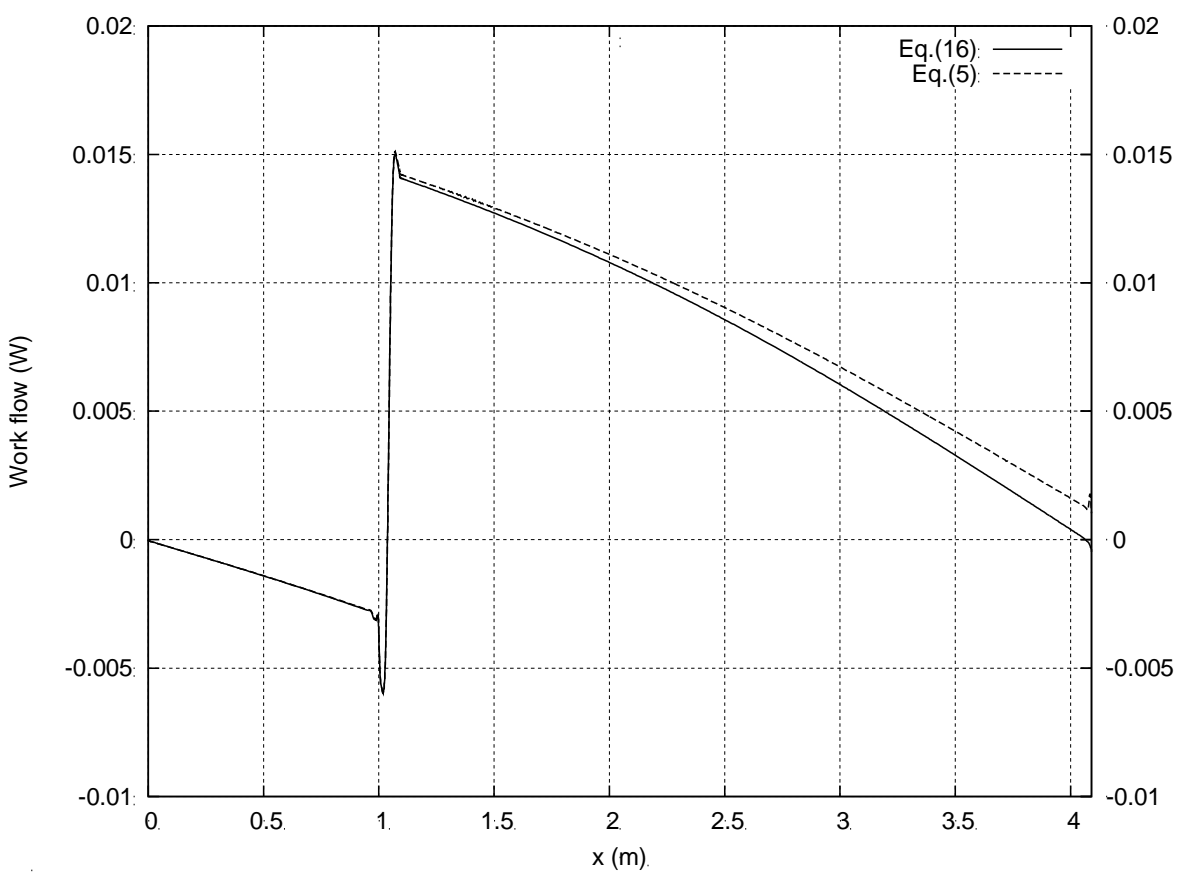

Fig. 8. Comparison of work flow and work source.

\subsection{Flow behavior in a temperature field}

Finally, the temperature field around the engine unit is investigated.

In the linear theory of Rott [8], it is assumed that the time-averaged temperature of the fluid in each section coincides with the wall temperature. However, because of heat transfer between the fluid and the solid wall, the averaged fluid temperature may possibly differ from the wall temperature. In fact, while the wall temperature of the HEX $(423.15 \mathrm{~K})$ is given as shown in Fig. 1, the fluid temperature in the CFD simulation is lower than the wall temperature. Figure 9 shows the time and section-averaged temperature distributions in the x-direction around the engine. White circles denote the results for each section along the device, and in the engine region ( $x=1.00$ to $1.09 \mathrm{~m}$ ), the temperature distribution for each channel is also shown in the graph. Each result corresponds to one of the center (black circles), bottom (white triangles) or top (black triangles) channels in the engine. The wall temperatures of the engine plates are 
plotted using a dotted line. There is a difference between the temperature of the fluid and that of the engine plates; specifically, the fluid temperatures in the bottom and top channels are much lower than that of the engine plates. This is because one side of each of these channels is set at room temperature $(298.15 \mathrm{~K})$. As a result, the effective temperature, when averaged over all engine channel sections (white circles), is further reduced when compared with the engine plate temperatures.

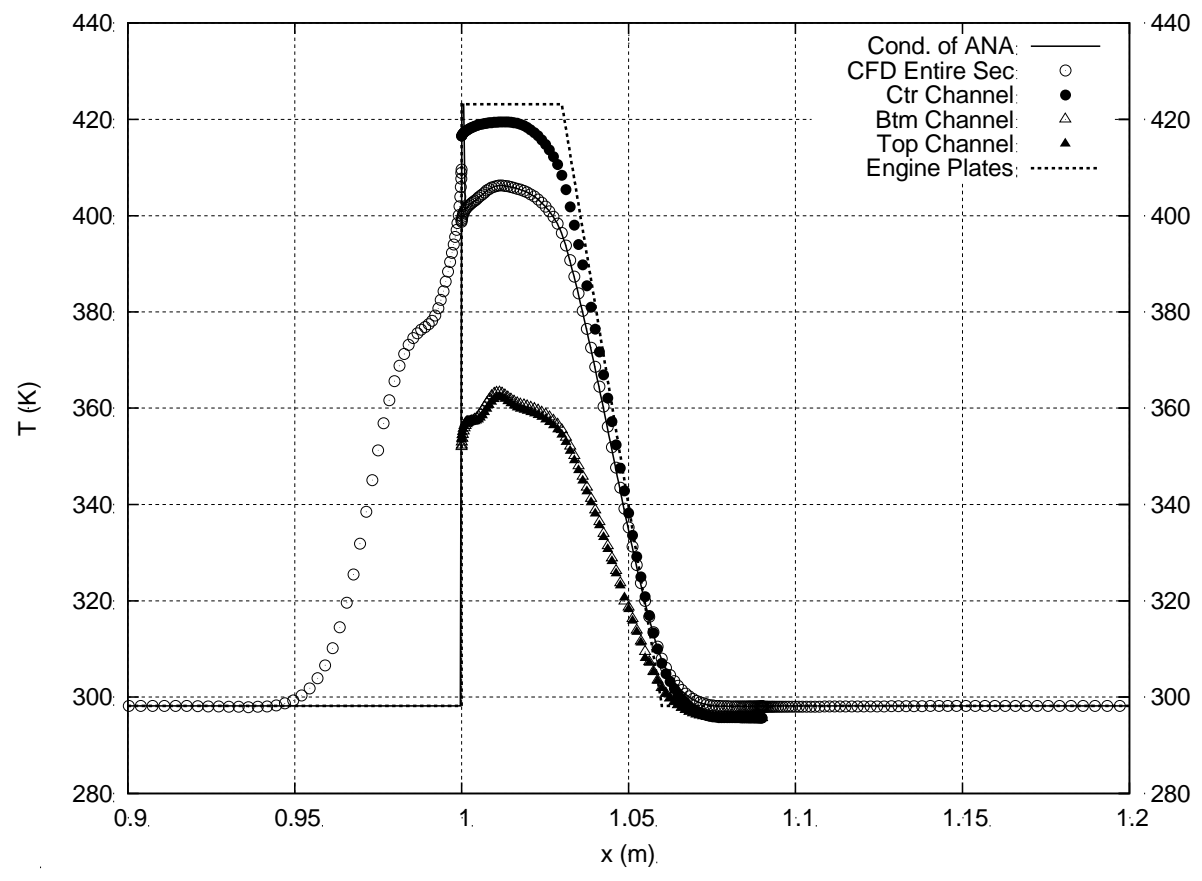

Fig. 9. Fluid temperature distribution along the axis.

On the other hand, a high-temperature region on the outside of the $\operatorname{HEX}(x=$ 0.95 to $1.00 \mathrm{~m}$ ) can also be observed. This is caused by leakage of heated air from the HEX; the area is called a thermal buffer region. The rapid increase in temperature at $x=1.0 \mathrm{~m}$ can be considered to be affected by the wall temperature at the engine plate extremity. The behavior of the heated air is visualized in Fig. 10. In this figure, the high temperature region of the thermal buffer is asymmetrical around the central line and deflects towards the upper wall. This is 
because the temperature is averaged over one period of an acoustic wave at most, despite the flow being disturbed by vortex generation.

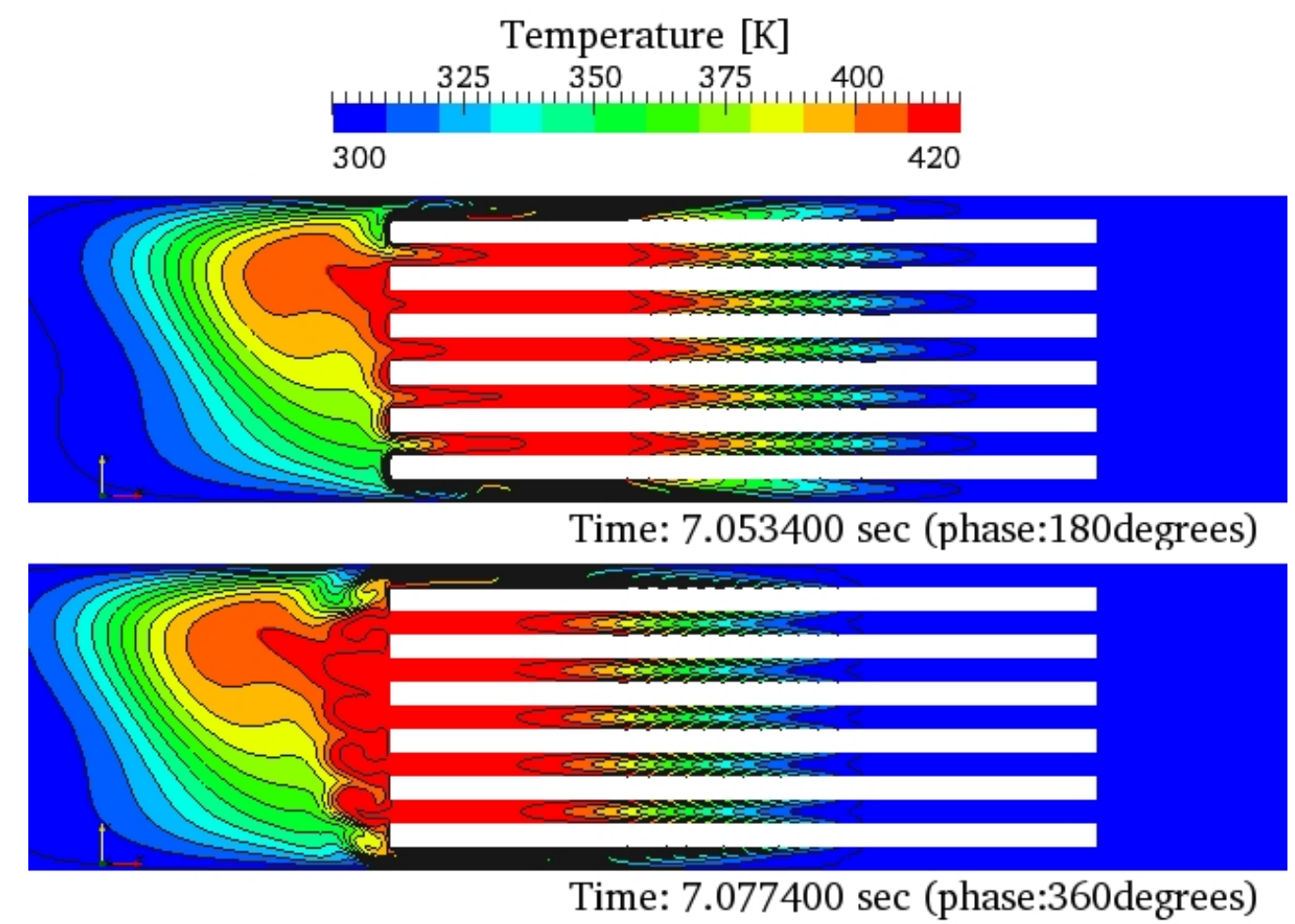

Fig. 10. Temperature distributions around the engine.

Next, to investigate the temperature field behavior in detail, the phase variations of the temperature are observed. The observed area is the central channel of the engine. The CFD results are also compared with the values that were predicted using Eq. (16) based on linear theory.

$T_{1}=\frac{1}{C_{p} \rho_{m}}\left(1-f_{\alpha}\right) p_{1}+\left(\frac{f_{\alpha}-f_{v}}{\left(1-\chi_{v}\right)(1-\sigma)}-\frac{1-f_{v}}{1-\chi_{v}}\right)\langle u\rangle_{r} \frac{1}{j \omega} \frac{\partial T_{m}}{\partial x}$

In Eq. (16), the temperature gradient in the $\mathrm{x}$-direction is taken from the temperature distribution on the center channel (black circles) shown in Fig. 9. 
Figure 11 shows the distribution within the central section of STK. The normalized temperature $\Theta$ must now be introduced:

$\Theta=\frac{T-T_{w}}{T_{H w}-T_{C w}}$,

where $T_{w}, T_{H w}$, and $T_{C w}$ are the wall temperatures of the local section, the HEX, and the CEX, respectively. As the graph shows, the CFD results closely follow those of the linear theory. This is because the temperature gradient in the $\mathrm{x}$-direction is almost uniform in this region.

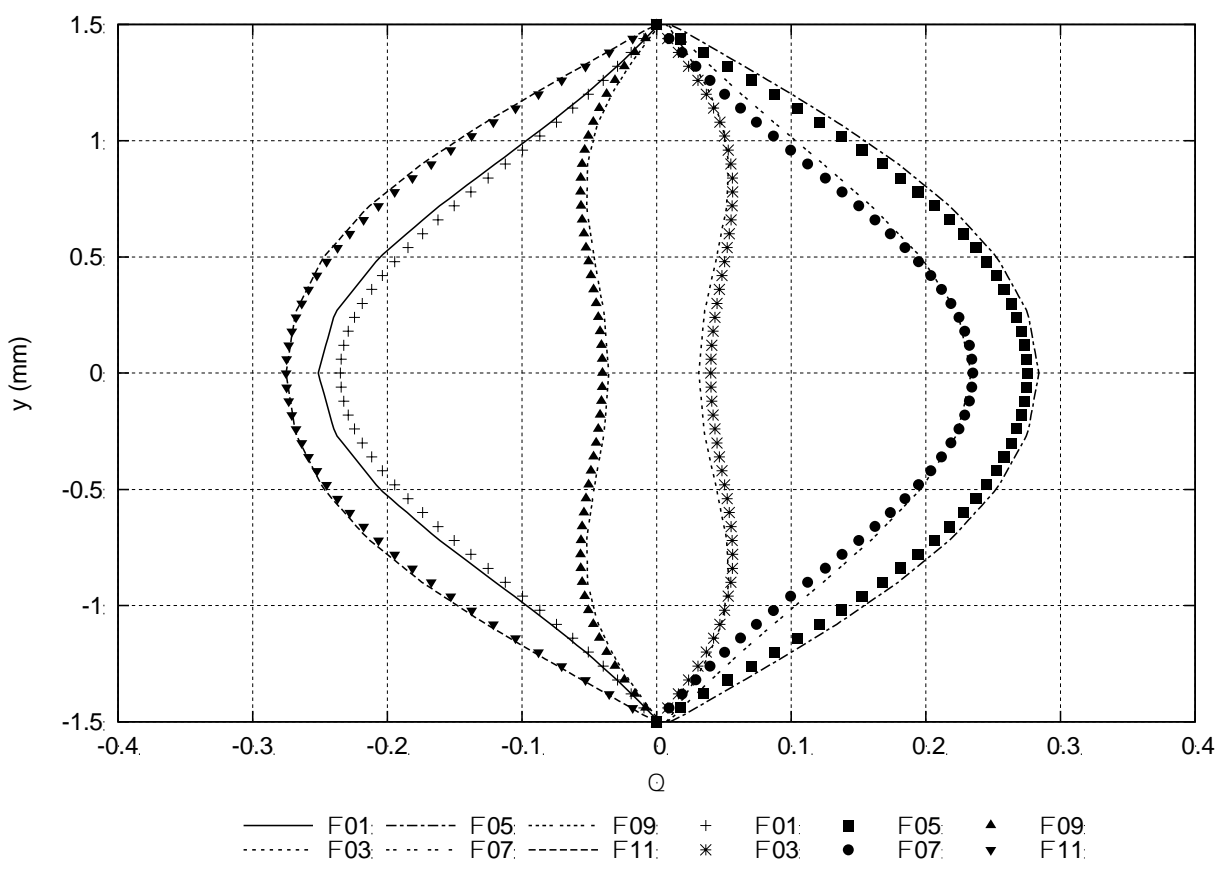

Fig. 11. Comparison of temperature distributions in the STK.

(Lines: CFD; symbols: linear analysis).

In contrast, at the boundary area between the heat exchanger (HEX and CEX) and the stack (STK), the situation is quite different. As shown in Fig. 12, the temperature oscillation from CFD does not agree with the results predicted using Eq. (16). The actual temperature oscillation in the 
CFD results is asymmetrical. In Eq. (16), the temperature gradient is assumed to be constant within the displacement amplitude of the local fluid element. In this case, the actual fluid element moves to an extent of $15 \mathrm{~mm}$, as shown in Fig. 3. When the temperature distribution shown in Fig. 9 is considered, the above assumption cannot be applied in this area. This means that the temperature gradient varies in the oscillatory cycle. 

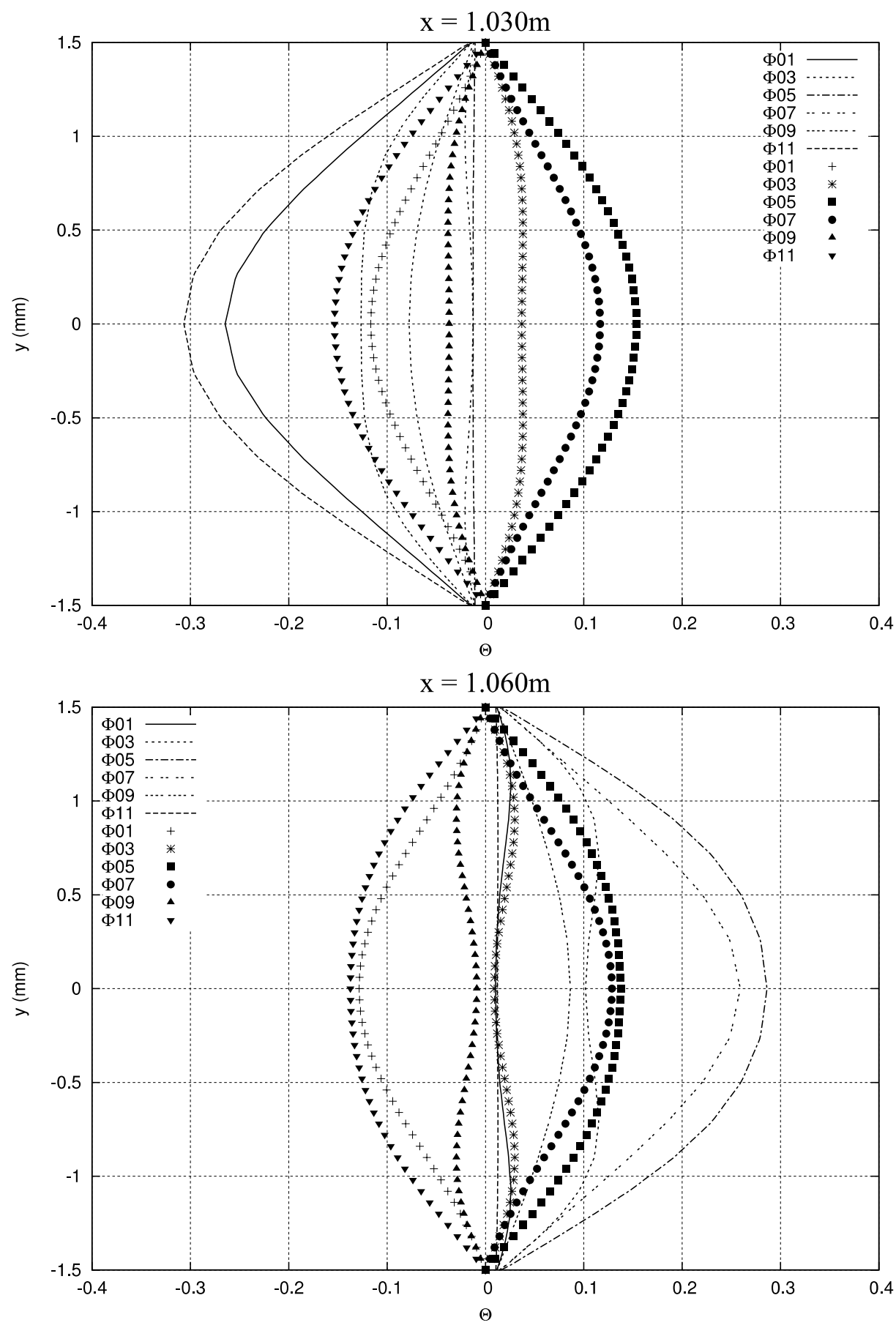

Fig. 12. Comparison of temperature distributions in HEX and CEX.

(Lines: CFD; symbols: linear analysis)

To improve this point, the convective term of Eq. (16) must therefore be modified. Here, it is assumed that the mean temperature gradient can be described using the linear transfer equation 
$\frac{\partial F}{\partial t}+u_{x} \frac{\partial F}{\partial x}=0, \quad F(x, t)=\frac{\partial T_{m}}{\partial x}$.

The solution to the above equation is:

$F(x, t)=\mathcal{F}\left(x-u_{x} t\right)$

Here, $u_{x} t$ corresponds to the fluid element displacement in the oscillatory flow.

In conventional linear theory, $\partial F / \partial x$ is equal to 0 within the length of the displacement order. This means that the value of $T_{1}$ calculated using Eq. (16) is unaffected by the temporal variation of the temperature gradient at a fixed point. Therefore, the temperature amplitude at phase $\varphi=\omega t$ can be calculated using Eq. (20).

$\mathcal{T}_{1}(\varphi)=\operatorname{Re}\left[T_{1} e^{j \varphi}\right]$

Here, $T_{1}$ is constant during the variation of $\varphi$.

In this modification, the following equation is used:

$\frac{\partial T_{m}}{\partial x}=\mathcal{F}\left(x-u_{x} t\right)=\mathcal{F}(x-\Delta \xi)$

$\Delta \xi=\operatorname{Im}\left[\frac{\langle u\rangle_{r}}{j \omega} e^{j \varphi}\right]$

The time variation of the temperature amplitude is then calculated using Eq. (23).

$\mathcal{T}_{1}(\varphi)=\operatorname{Re}\left[T_{1}(\varphi) e^{j \varphi}\right]$

Using this modification, the analytical temperature distribution then approaches that of the CFD results, as shown in Fig. 13. This implies that the convective term used above for temperature behavior in the heat exchanger is substantial. 

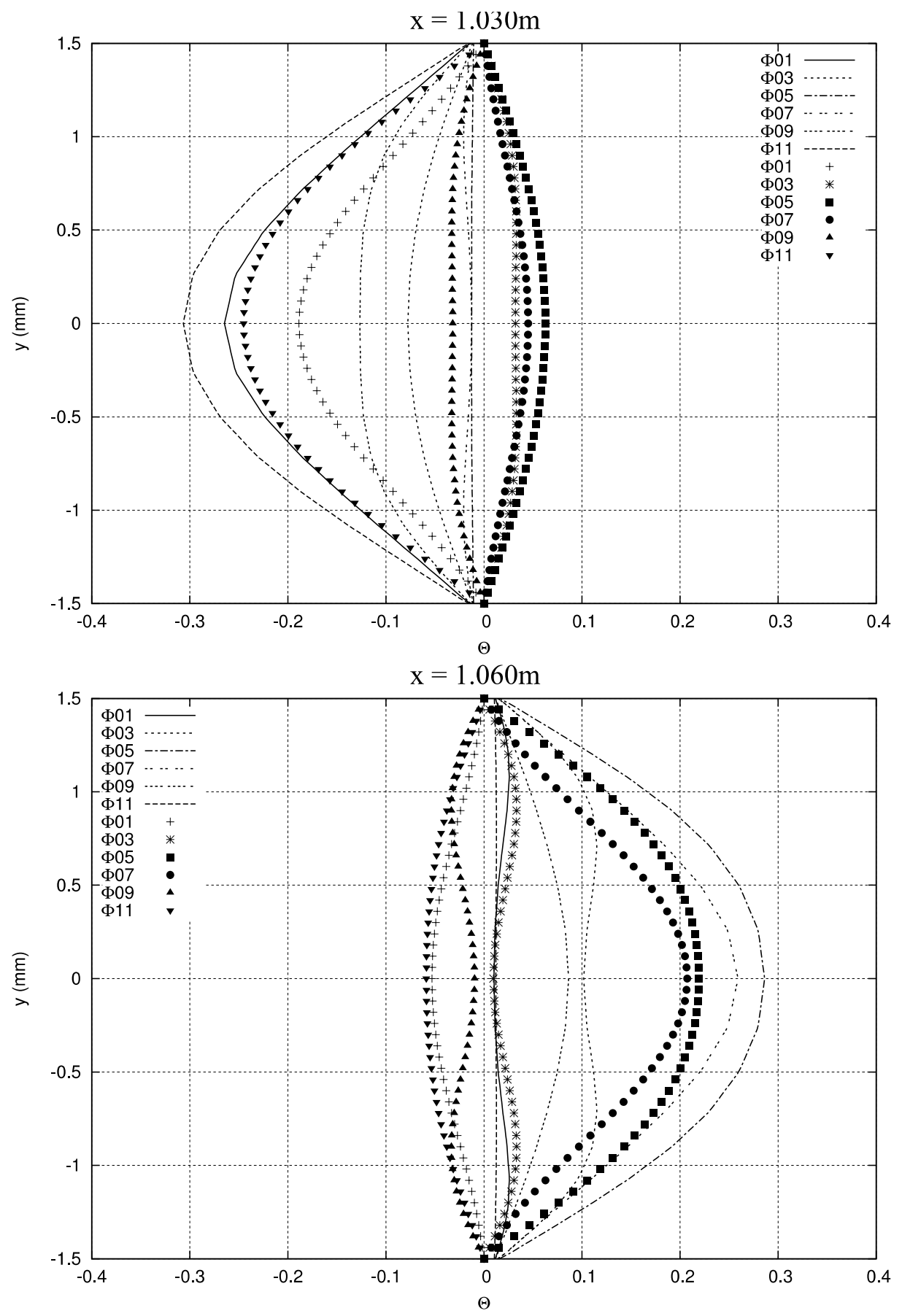

Fig. 13. Comparison of modified temperature distributions in HEX and CEX.

(Lines: CFD; symbols: linear analysis). 


\section{Conclusions}

Using CFD techniques, numerical simulations of the oscillatory flow within a thermoacoustic device were performed. This straight-type device generates a standing acoustic wave. The CFD results were also compared with those generated using linear theory. From a discussion of these results, the following conclusions are drawn.

- Injection of an acoustic signal that acts as a trigger pulse into the device allows selfsustained thermoacoustic oscillation to be reproduced in the CFD simulations.

- The dissipation terms in the CFD simulations were assigned to the thermoacoustic properties from linear theory, and each property was compared for differences between CFD and linear analysis. From this comparison, it was found that the estimated acoustic power obeys linear theory, although non-linear effects appear near the engine plate extremities. This occurs because these phenomena are limited to narrow regions under the conditions used.

- The temperature oscillation shows a strongly asymmetrical structure within the engine, unlike that in the resonance tube region. This is due to the non-uniformity of the temperature gradient within the engine. Additionally, by introducing a phase transfer to estimate the temperature gradient and then modifying conventional linear theory, it would be possible to improve temperature field predictions within the heat exchanger.

\section{Acknowledgments}

This work was supported by the Japan Science and Technology Agency through the Advanced Low Carbon Technology Research and Development Program. 


\section{References}

[1] G.W. Swift, Thermoacoustic Engines, J. Acoust. Soc. Am. 84 (1988) 1145.

[2] S.N. Backhaus, G.W. Swift, A thermoacoustic Stirling heat engine, Nature 399 (1999) 335338.

[3] R. Kirchhoff, Über den Einfluss der Wärmeleitung in einem Gase auf die Schallbewegung (English: On the influence of heat conduction in a gas on sound propagation), Pogg. Ann. 134 (1868) 177-193.

[4] J.W.S. Rayleigh, The Theory of Sound, vol. II, Dover, New York, 1945, 319-326.

[5] C. Zwikker, C.W. Kosten, Sound Absorbing Materials, Elsevier, Amsterdam, 1949, 25-52.

[6] D.E. Weston, The theory of the propagation of plane sound waves in tubes, Proc. Phys. Soc. London B.66 (1953) 695-709.

[7] S. Iberall, Attenuation of oscillatory pressures in instrument lines, J. Res. Nat. Bur. Stand. 45 (1950) 85-108.

[8] N. Rott, Damped and thermally driven acoustic oscillations in wide and narrow tubes, $Z$. Angew. Math. Phys. 20 (1969) 230-243.

[9] N. Rott, Thermally driven acoustic oscillations, part III: Second-order heat flux, Z. Angew. Math. Phys. 26 (1975) 43-49.

[10] H. Tijdeman, On the propagation of sound waves in cylindrical tubes, J. Sound Vib. 39 (1975) 1-33.

[11] B. Ward, J. Clark, G. Swift, DeltaE: Design environment for low amplitude thermoacoustic engines: User guide and tutorial, Los Alamos National Laboratory, LA-CC-93-8, 1996. 
[12] A. Piccolo, G. Pistone, Estimation of heat transfer coefficients in oscillating flows: The thermoacoustic case, Int. J. Heat Mass Transfer, 49 (2006) 1631-1642.

[13] A. Piccolo, Numerical computation for parallel plate thermoacoustic heat exchangers in standing wave oscillatory flow, Int. J. Heat Mass Transfer, 54 (2011) 4518-4530.

[14] J.A. de Jong, Y.H. Wijnant, A. de Boer, A one-dimensional heat transfer model for parallel-plate thermoacoustic heat exchangers, J. Acoust. Soc. Am. 135 (2014) 1149.

[15] R.S. Wakeland, R.M. Keolian, Effectiveness of parallel-plate heat exchangers in thermoacoustic devices, J. Acoust. Soc. Am., 115 (2004) 2873.

[16] L. Shi, X. Mao, A.J. Jaworski, Application of planar laser-induced fluorescence measurement techniques to study the heat transfer characteristics of parallel-plate heat exchangers in thermoacoustic devices, Mas. Sci. Technol. 21 (2010) 115405.

[17] L. Shi, Z. Yu, A.J. Jaworski, Application of laser-based instrumentation for measurement of time-resolved temperature and velocity fields in the thermoacoustic system, Int. J. Therm. Sci. 49 (2010) 1688-1701.

[18] A.J. Jaworski, A. Piccolo, Heat transfer processes in parallel-plate heat exchangers of thermoacoustic devices - numerical and experimental approaches, Appl. Therm. Eng. 42 (2012) $145-153$.

[19] Z. Yu, X. Mao, A.J. Jaworski, Experimental study of heat transfer in oscillatory gas flow inside a parallel-plate channel with imposed axial temperature gradient, Int. J. Heat Mass Transfer 77 (2014) 1023-1032.

[20] T. Zhao, P. Cheng, A numerical solution of laminar forced convection in a heated pipe subjected to a reciprocating flow, Int. J. Heat Mass Transfer 38 (1995) 3011-3022. 
[21] T. Zhao, P. Cheng, Oscillatory heat transfer in a pipe subjected to a laminar reciprocating flow, ASME J. Heat Transfer 118 (1996) 592-598.

[22] N. Cao, J.R. Olson, G.W. Swift, S. Chen, Energy flux density in a thermoacoustic couple, J. Acoust. Soc. Am. 99 (1996) 3456.

[23] H. Ishikawa, D.J. Mee, Numerical investigations of flow and energy fields near a thermoacoustic couple, J. Acoust. Soc. Am. 111 (2002) 831.

[24] D. Marx, Ph. Blanc-Benon, Numerical calculation of the temperature difference between the extremities of a thermoacoustic stack plate, Cryogenics 45 (2005) 163-172.

[25] F.A.Z. Mohd, A.J. Jaworski, Oscillatory flow and heat transfer within parallel-plate heat exchangers of thermoacoustic systems, Proc. World Congress on Eng. 2013, vol. III (2013).

[26] C.C. Hantschk, D. Vortmeyer, Numerical simulation of self-excited thermoacoustic instabilities in a Rijke tube, J. Sound Vib. 277 (1999) 511-522.

[27] J.A.L. a Nijeholt, M.E.H. Tijani, S. Spoelstra, Simulation of a traveling wave thermoacoustic engine using computational fluid dynamics, J. Acoust. Soc. Am. 118 (2005) $2265-2270$.

[28] F. Zink, J. Vipperman, L. Schaefer, CFD simulation of a thermoacoustic engine with coiled resonator, Int. Commun. Heat Mass 37 (2010) 226-229.

[29] G. Yu, W. Dai, E. Luo, CFD simulation of a $300 \mathrm{~Hz}$ thermoacoustic standing wave engine, Cryogenics 50 (2010) 615-622.

[30] K. Fujimoto, K. Fujii, Study on the automated CFD analysis tools for conceptual design of space transportation vehicle, Proc. $5^{\text {th }}$ ASME/JSME Joint Fluid Eng. Conf. (2007) FEDSM 2007-37128. 
[31] A. Jameson, E. Turkel, Implicit schemes and LU decompositions, Math. Comp. 37 (1981) 385-397.

[32] E. Shima, K. Kitamura, Parameter free, simple, low-dissipation AUSM-family scheme for all speeds, AIAA J. 49 (2011) 1693-1709.

[33] Z.J. Wang, A quadtree-based adaptive Cartesian/quad grid flow solver for Navier-Stokes equations, Comput. Fluids 27 (1998) 529-549.

[34] Y. Ueda, C. Kato, Stability analysis of thermally induced spontaneous gas oscillations in straight and looped tubes, J. Acoust. Soc. Am. 124 (2008) 851-858.

[35] A. Tominaga, Thermodynamic aspects of thermoacoustic theory, Cryogenics, 35 (1995) $427-440$.

\author{
Nomenclature \\ $\rho \quad$ density \\ $\rho_{m} \quad$ mean (time-averaged) density \\ $u_{i} \quad$ flow velocity vector \\ $\langle u\rangle_{r} \quad$ velocity amplitude at cross-section \\ $p \quad$ absolute pressure \\ $p_{m} \quad$ mean (time-averaged) pressure \\ $p_{1} \quad$ pressure amplitude at cross-section \\ $\varphi \quad$ phase of acoustic wave \\ $\varphi_{p u} \quad$ phase difference between pressure and velocity \\ $T \quad$ absolute temperature \\ $T_{m} \quad$ mean (time-averaged) temperature
}




\begin{tabular}{|c|c|}
\hline$T_{1}$ & temperature amplitude at cross-section \\
\hline$T_{w}$ & wall temperature \\
\hline$T_{H w}, T_{C w}$ & wall temperatures of $\mathrm{HEX}$ and CEX, respectively \\
\hline$n_{i}$ & normal vector of surface element \\
\hline$t$ & time \\
\hline$\Delta t$ & period of acoustic wave \\
\hline$f$ & frequency of acoustic wave \\
\hline $\mathcal{V}$ & volume element of control volume \\
\hline$S$ & surface element of control volume \\
\hline$A$ & area of cross-section \\
\hline$R$ & gas constant \\
\hline$\tau_{i j}$ & shear stress tensor \\
\hline$q_{j}$ & heat flux \\
\hline$\mu$ & viscosity \\
\hline$v$ & kinetic viscosity \\
\hline$\lambda$ & thermal conductivity \\
\hline$\alpha$ & thermal diffusivity \\
\hline$\gamma$ & specific heat ratio \\
\hline$\sigma$ & Prandtl number \\
\hline$\delta_{i j}$ & Kronecker delta \\
\hline$j$ & imaginary unit \\
\hline$\omega$ & angular frequency \\
\hline & thermoacoustic function \\
\hline
\end{tabular}




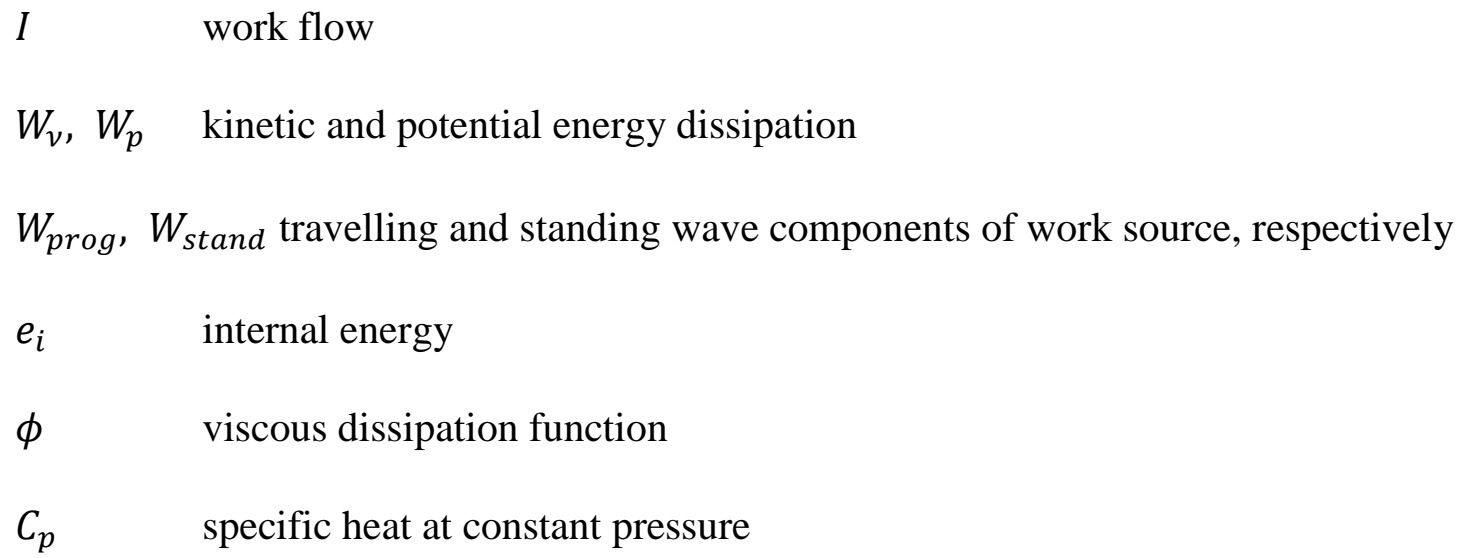

